Erschienen in: Stickel, Gerhard (Hrsg.): Stilfragen. - Berlin, New York: de Gruyter, 1995. S. 386-391. (Institut für deutsche Sprache. Jahrbuch 1994)

\author{
WILLY SANDERS
}

\title{
Stil und Stilistik
}

„Stil ist einer der schwierigsten Begriffe, die in diesem Buch gebraucht werden."

D. Crystal, 1993, S. 66

\section{Stilfragen}

Ein leicht anzügliches Wort sagt, es bedürfe nur der Teilnahme an einem StilkongreB, um zu wissen, wie man sich die Babylonische Sprachverwirrung vorzustellen habe. Richtig daran ist: Stil und Stilistik werden, was Definition, Terminologie, Prinzipien, Methoden usw. angeht, derart kaleidoskopisch bis kontrovers behandelt, daB sich an der Einheitlichkeit des Stilbegriffs füglich Zweifel einstellen können. Man braucht nur den Sammelband von H.U. Gumbrecht/K.L. Pfeiffer (1986) zur Hand zu nehmen, und schon irrt man zwischen literaturwissenschaftlichen und kunsthistorischen, soziologischen und kulturgeschichtlichen, ja lebensweltlichphilosophischen Stilauffassungen hin und her, die sich allenfalls unter dem sehr weiten Himmel einer universalen Stilsemiotik vereinen lassen. Der Stilbegriff, heißt es dort dezidiert, habe sich in seiner ursprünglichen sprachlich-literarischen Form verschlissen, erziele dafür jedoch in heutiger Zeit Anwendungszugewinne in vielen anderen Bereichen. Zu längst bekannten Stichwörtern wie „offener Stil” oder „Stilpluralismus” gesellt sich die Vorstellung einer „Ubiquität” des Stils, der überall dort - in einer Art Nothelferfunktion - einspringe, "wo andere Begriffe zu versagen drohen" (K.L. Pfeiffer, ebd. S. 693f. und 711). Auch sonst geht, dieses Forum eingeschlossen, nicht selten ein Stilskeptizismus um, dem man nur mit der kühnen Feststellung U. Püschels (1991, S. 65) begegnen kann: "Wenn es den Begriff des Sprachstils nicht gäbe, so müßte er unbedingt erfunden werden."

Die folgenden Bemerkungen beruhen auf Vorarbeiten zu einer 'Studienbibliographie Stil und Stilistik', die im Auftrag des Instituts für deutsche Sprache entsteht. Nur auswahlweise und bestenfalls andeutend geben sie einige Beobachtungen wieder, die aus der Durchsicht von gut anderthalbtausend Titeln stilistischen Gehalts resultieren und - Postskriptum - mit vielen Vorträgen der Tagung erstaunlich korrespondieren.

Vorweg ein doppelter Pauschaleindruck: Quantitativ stammt, erstens, die Mehrzahl der bibliographisch erfaBten Stilpublikationen aus den letzten zwanzig Jahren. Das ist nicht Ergebnis einer Auswahl nach Aktualitätsgesichtspunkten, sondern spiegelt den bemerkenswerten Aufschwung, den die Sprachstilistik seit den 60er Jahren genommen hat, und zwar im 
Gefolge des damaligen Höhenflugs der modernen Linguistik, nachdem bis dahin die literaturorientierte Stilbetrachtung absolut vorherrschte (insofern ist es kein Widerspruch, wenn der Literaturwissenschaftler konstatiert, es sei still geworden um den Stilbegriff, der seine große Zeit hinter sich habe; J. Anderegg, in diesem Band S. 115). Qualitativ läBt sich, zweitens, nach einer Phase starken Theoretisierens gerade in letzter Zeit eine Tendenz zu pragmatischen Anschauungen, verbunden mit praktischen Veranschaulichungen, feststellen. Ein schönes Beispiel für solche Fachmetaphorik ist das seit 1980 durch das stilistische Schrifttum und mehrmals auch durch den Vortragssaal geisternde "Chamäleon Stil”.

\section{Die Stil-Frage}

Nicht nur zur Einleitung von Stiltagungen (H.-M. Gauger, S. 7-26; vgl. ders. 1991, 1993), sondern auch am Anfang unzähliger Stilbücher und artikel wird gefragt: „Was ist (eigentlich) Stil?” - die Stil-Frage schlechthin. Die Antworten verbleiben entweder im Topischen, wie W.G. Müller (1981) des näheren dargelegt hat; beispielsweise das zeitlose, auch jetzt wieder aktuelle Diktum des Grafen Buffon, der Stil sei der Mensch selbst ("le style est l'homme même"). Oder sie sind einerseits, gebunden an spezielle Konzeptionen wie „Stil als Abweichung von der Norm”, zu eng, um Allgemeingültigkeit beanspruchen zu können; andrerseits aber, wenn sie ihr Heil in synthetisierenden Umschreibungen suchen, zu weit, um praktikabel zu sein. Vielleicht ist "Stil" gleich anderen sprachwissenschaftlichen Zentralbegriffen - „Wort”, "Satz”, „Text" usw. - gar nicht exakt definierbar? Ein ebenso beliebter Aufhänger wie die Stil-Frage ist übrigens seine Wortgeschichte, die vom antik-mittelalterlichen "Schreibgriffel”, dann "Schreibart”, zum neuzeitlichen „Stil” führt: vielen erscheint der lateinische stilus in der Hand offenbar sicherer als ein moderner Stilbegriff auf dem Dach.

Die enorme Wichtigkeit des Stils für unseren Umgang mit Sprache wird kaum bestritten, indes bereitet eine klare Abgrenzung von eben dieser Sprache Mühe. In der gegenwärtigen Fachdiskussion hat das problematische Verhältnis von matter und manner, wie es englisch heißt, Ausdruck gefunden in der "vorwissenschaftlichen”, aber anschaulichen Unterscheidung des WAS und des WIE von Sprachäußerungen: das "Was” als Sachgehalt des Geäußerten, das „Wie” als seine sprachlich-stilistische Äußerungsform. Hochinteressant $z u$ verfolgen, wie sich während der letzten zehn Jahre in kleinen, oft beiläufigen Diskussionsschritten eine Entwicklung vollzieht von der Auffassung eines völlig bedeutungsindifferenten WIE über seine Sicht als Einstellungen, Haltungen, Wertungen ausdrückend bis zur heutigen Annahme einer eigenen „stilistischen 
Bedeutung", die direkten Einfluß auf das WAS hat und am Sinn der Äußerung "maßgeblich beteiligt" ist (Thieberger 1992, S. 52). Doch geht es nicht nur um das mittlerweile fast omnipräsente WAS und WIE allein, ihren so oder so formulierten Zusammenhang; vielmehr wird mit weiteren Fragen, etwa nach dem WARUM und WOZU, der bloBe Bedeutungsaspekt um wichtige Gesichtspunkte ergänzt wie Stilabsicht und Stilwirkung. Zugleich erschlieBt sich der Hintergrund jenes auf alte, rhetorische Muster zurückgehenden Katalogs von W-Fragen (WER, WAS, WEM usw.; vgl. Stolt 1984), die als solche den kommunikativen Grundcharakter des Stils offenlegen.

\section{Fragestellungen}

Manche Stilprobleme darf man unterdessen als gelöst ansehen, andere stehen weiterhin zur Diskussion (im Überblick Sowinski 1991). Während mit der kommunikativ-pragmatischen Fundierung der Stilistik im Rahmen von Sprachhandlungstheorie und Semiotik eine tragfähige Grundlage geschaffen scheint (vgl. Sandig 1978, 1986), irritiert - immer vom Bibliographischen her gesehen - neben anderen, gut dokumentierten Stilkonzeptionen wie Individualstil, Funktionalstilistik oder Stilstatistik die keineswegs neue Auffassung von „Stil als Wahl”: genauer die Auswahl aus synonymischen, äquivalenten, konkurrierenden usw. Sprachmöglichkeiten - eine Auffassung, die vielfach geradezu als stilistische Prämisse, d.h. unerläBliche Voraussetzung für die Existenz des Stilphänomens angesehen wird. Um so mehr muB das fast vollständige Fehlen spezieller neuerer Arbeiten auffallen (einschlägig nur Agricola 1957; Milic 1971). Die Auseinandersetzung mit der Selektionstheorie spielt sich nahezu ausschlieBlich in Handbuchartikeln und Gesamtdarstellungen ab, die ihr mehr oder weniger Raum widmen, und sie ist zudem gekennzeichnet durch die zwiespältige Argumentationsfigur des „Zwar - aber”. Umstritten sind vor allem die notwendigerweise anzunehmenden Selektionsbeschränkungen, der Selektionsbegriff im stilistischen Analyseverfahren und der Bewußtheitsgrad der Stilwahl, inwieweit nämlich etwas intentional „zum Ausdruck gebracht wird” oder unreflektiert „zum Ausdruck kommt" (nach Keller 1977, S. 15). Klärung täte zweifellos not.

„Tendenzen der linguistischen Stilforschung". wird man nach der umfassenden Übersicht von B. Sandig (S. 27-61) nicht mehr aufzählen, wohl aber seien einige stilistische Problemfelder angesprochen. Bibliographisch defizitäre Bereiche bilden u.a. die Stilgeschichte, eingeschlossen das Phänomen des Stilwandels (vgl. G. Lerchner, S. 94-114), und ein Konzept von Zeit- und Epochenstilen, das nicht literaturgebunden ist; eine parallel zu Interlinguistik, Interkultureller Kommunikation usw. 
zu postulierende „Interlinguale Stilistik”, die in engster Verbindung mit einer Übersetzungsstilistik zukunftsträchtig erscheint; nicht zuletzt aber die noch junge Makrostilistik: Nachdem schon Asmuth (1974, S. 67f.) vor der "Froschperspektive" der traditionellen Stilistik gewarnt hatte, die nur "die grammatisch, lexisch und phonetisch beschreibbare, im Grunde sekundäre Mikrostuktur" berücksichtige, war es die Moskauer Stilforscherin E. Riesel, die erstmals eine systematische Unterscheidung von Mikro- und Makrostilistik vorschlug - letztere ausdrücklich als damals „noch wenig bearbeitetes Neuland” (Riesel/Schendels 1975, S. 11f.). Seither sind zögernd erste Arbeiten erschienen, in denen sich freilich die angestrebte makrostilistische "Adlerperspektive", so Püschel (1991, S. 64), bestenfalls umrißhaft andeutet. Zumal vor dem Horizont eines kommunikativ-pragmatischen Stilbegriffs stellen die Zusammenschau der "globalen" Textorganisation mit den sie konstituierenden Einzelelementen, die Wechselbeziehung also zwischen dem Ganzen und seinen Teilen, ebenso wie eine Klärung des problematischen "Denkstils" (Sanders 1990, S. 23ff.) vordringliche Forschungsaufgaben dar.

Bleibt noch ein kurzer Blick auf die sog. Praktische (normative, präskriptive) Stilistik, das weite, durchweg populär beackerte Feld von Sprachkritik und Stillehre, denen G. Antos (SchluBvortrag, S. 355-377) ihren Ort im Rahmen einer landläufigen "Laien-Linguistik" zugewiesen hat. Es ist sicher nicht damit getan, über solche „Sprachkritikastereien” (Sanders 1992) zu lamentieren; vielmehr gilt es, auf der Grundlage linguistisch fundierter Stilkritik und Stilbewertung eine wissenschaftliche Stildidaktik zu entwickeln, die sich ernsthaft mit den Möglichkeiten praktischer Stillehre auseinandersetzt und auch nicht vor der Herausforderung des bislang arg vernachlässigten Lehrbuchs zurückschreckt. Zur Bezeichnung dieses Bereichs stilistischer Textgestaltung, ihrer Mittel und Vermittlung, bietet sich der im Soziologischen beheimatete Begriff der "Stilisierung" an, der neuerdings - wie die Titel von Neuland/Bleckwenn (1991) und Hinnenkamp/Selting (1989) zeigen - nicht immer, aber immer öfter auch sprachwissenschaftlich für Stilgebung im weitesten Sinne steht: das „Stilherstellen”, wie es technizistisch heißt.

\section{Nach wie vor frag-würdig?}

Um am SchluB den Bogen zurück zum Anfang der Tagung zu schlagen: Stil sei einer der schwierigsten Begriffe überhaupt, zitierte B. Spillner (hier S. 64) den englischen Sprachenzyklopädisten D. Crystal - das wird vermutlich zum geflügelten Wort künftiger Stilistik werden. H.-M. Gauger (S. 21) hat sich, Buffon variierend, dieser Schwierigkeit des Stilbegriffs geschickt durch eine so nur französisch mögliche Pointe entzogen: 
„Le style (Stil/Griffel), c'est le style (Griffel/Stil) même." Man kann dies deutsch auf die Spitze (französisch pointe) treiben, indem man Karl Philipp Moritz zitiert; in seinen 'Vorlesungen über den Stil' (1793/94; nach R. Campe 1991, S. 75) steht, wie immer das zu interpretieren sein mag: "Der Stil ist - der Stil".

\section{Literatur}

Agricola, Erhard (1957): Fakultative sprachliche Formen. Gedanken zur grammatischen Fundierung der Stilkunde. In: Beiträge zur Geschichte der deutschen Sprache und Literatur/Halle 79, Sonderband für Theodor Frings, S. 43-76.

Asmuth, Bernhard/Berg-Ehlers, Luise (1974): Stilistik. Düsseldorf. (Grundstudium Literaturwissenschaft. Hochschuldidaktische Arbeitsmaterialien, Bd. 5).

Campe, Rüdiger (1991): Die zwei Perioden des Stils. In: Comparatio 2, S. 73-101.

Crystal, David (1993): Die Cambridge Enzyklopädie der Sprache. Frankfurt a.M.

Gauger, Hans-Martin (1991): Zur Frage des Stils - etymologisch gesehen. In: Comparatio 2, S. 3-16.

Gauger, Hans-Martin (1993): Stilfragen. In: Merkur 47, S. 494- 507.

Gumbrecht, Hans Ulrich/Pfeiffer, Karl Ludwig (Hg.) (1986): Stil. Geschichten und Funktionen eines kulturwissenschaftlichen Diskurselements. Frankfurt a.M. (suhrkamp taschenbuch wissenschaft).

Hinnenkamp, Volker/Selting, Margret (Hg.) (1989): Stil und Stilisierung. Arbeiten zur interpretativen Soziolinguistik. Tübingen. (Linguistische Arbeiten, 235).

Keller, Rudi (1977): Kollokutionäre Akte. In: Germanistische Linguistik 1-2/77, S. 3-50.

Milic, Louis T. (1971): Rhetorical Choice and Stylistic Option. The Conscious and Unconscious Poles. In: Chatman, Seymour (Hg.): Literary Style. A Symposium. London/New York. S. 77-88.

Müller, Wolfgang G. (1981): Topik des Stilbegrifts. Zur Geschichte des Stilverständnisses von der Antike bis zur Gegenwart. Darmstadt. (Impulse der Forschung, 34).

Neuland, Eva/Bleckwenn, Helga (Hg.) (1991): Stil - Stilistik - Stilisierung. Linguistische, literaturwissenschaftliche und didaktische Beiträge zur Stilforschung. Frankfurt a.M./Bern/New York/Paris.

Püschel, Ulrich (1991): Praktische Stilistiken - Ratgeber für gutes Deutsch? In: Neuland/Bleckwenn (Hg.) (1991), S. 55-68.

Riesel, Elise/Schendels, Eugenie (1975): Deutsche Stilistik. Moskau.

Sanders, Willy (1990): Gutes Deutsch - besseres Deutsch. Praktische Stillehre der deutschen Gegenwartssprache. 2. Aufl. Darmstadt. 
Sanders, Willy (1992): Sprachkritikastereien und was der „Fachler” dazu sagt. Darmstadt.

Sandig, Barbara (1978): Stilistik. Sprachpragmatische Grundlegung der Stilbeschreibung. Berlin/New York. (de Gruyter Studienbuch).

Sandig, Barbara (1986): Stilistik der deutschen Sprache. Berlin/New York. (Sammlung Göschen, 2229).

Sowinski, Bernhard (1991): Stilistik. Stiltheorien und Stilanalysen. Stuttgart (Sammlung Metzler, 263).

Stolt, Birgit (1984): Pragmatische Stilanalyse. In: Spillner, Bernd (Hg.) (1984): Methoden der Stilanalyse. Tübingen. S. 163-173.

Thieberger, Richard (1992): Das Wie und das Was. In: Hardin, James/Jungmayr, Jörg (Hg.) (1992): „Der Buchstab tödt - der Geist macht lebendign. Festschrift zum 60. Geburtstag von Hans-Gert Roloff. Bd. I. Bern/Berlin/Frankfurt a.M./New York/Paris/Wien. S. 47-54. 